\title{
Sonodynamic Therapy for Recurrent Plexiform Neurofibroma: A Case Report
}

Ying Ji*

Beijing Shengkang Clinic, Beijing, China

*Corresponding author: Ying Ji, Beijing Shengkang Clinic, Beijing, China, Tel: 0118613801202542; E-mail: doctorjiying@sina.com

Received: 29 Jul, 2020 | Accepted: 12 Aug, 2020 | Published: 18 Aug, 2020

Citation: Ji Y (2020) Sonodynamic Therapy for Recurrent Plexiform Neurofibroma: A Case Report. J Clin Case Stu 5(3): dx.doi.org/10.16966/24714925.204

Copyright: $\odot 2020 \mathrm{Ji}$ Y. This is an open-access article distributed under the terms of the Creative Commons Attribution License, which permits unrestricted use, distribution, and reproduction in any medium, provided the original author and source are credited.

\begin{abstract}
Neurofibromatosis (NF 1) is an autosomal genetic disease caused by mutations in the 17q11.2 gene, called the NF1 gene, with an incidence rate of $1 / 2500$. Characteristics of the disease are the growth of Cafe au lait spots, freckles on the jaw and armpits, and neurofibromas growing along nerve fibers. Plexiform neurofibromatosis usually involves multiple nerves, clinically manifested as swelling and irregular skin lumps involving the surrounding hoof tissue and skin folds in a shape similar to a "bags of worms." The primary treatment methodology has been surgery, but the treatment effects remain unsatisfactory, with frequent postoperative recurrences and inability to lead to full recovery from the disease even after multiple operations. We have used Sonodynamic Therapy (SDT), a new treatment technique in a case of recurrent plexiform neurofibroma following repeatedly surgery. In this report, we discuss the significance of a new treatment technology for recurrent neurofibromatosis.
\end{abstract}

Keywords: Sonodynamic therapy; Plexiform neurofibroma; Neurofibromatosis

Abbreviations: SDT: Sonodynamic Therapy; NF: Neurofibromatosis; CUSG: Color Ultrasonic Diagnostic Apparatus

\section{Case Report}

A 20-year-old female patient arrived at her first treatment hospital in April 2013 to treat swelling in her left foot that caused difficulties with walking. Examinations revealed a mass on her left foot painful during palpations; no other abnormalities were found. The left foot mass was removed by surgery. Its size was $1.5 \mathrm{~cm} \times 1.5 \mathrm{~cm}$, with a grayish white appearance and crisp texture. Pathological examination revealed a large number of fusiform and neuroperiosteal cells and immunohistochemistry (HI13-3610): S100+, bc1-2+, CD99+, epithelial cells EMA+, CD34+, AE1/AE3-, a-SMA-, desmin-. Molecular Pathology (MP13-1324) was found using the FISH method to be $\mathrm{t}(18 \mathrm{q} 11.2)(\mathrm{SYT}):(-)$. The diagnosis was mixed schwannoma/ neurasthema, with tendencies to plexiform schwannomas. Postsurgery, the patient experienced a smooth recovery.

In August 2016, due to a recurrence of the mass and pain in left foot in the area of the original surgical procedure, the patient returned to her first treatment hospital for treatment. Examination revealed surgical scars on the sole of the left foot and a $1 \mathrm{~cm} \times 1 \mathrm{~cm}$-sized mass accompanied by marked tenderness detected by Color Ultrasonic Diagnostic Apparatus (CUSG), also known as color doppler ultrasound, of probe frequency $12 \mathrm{MHz}$. No other abnormalities were found. The diagnosis was thus recurrence of plexiform schwannoma. The tumor was surgically removed again and postoperative recovery was smooth.

In March 2019, the left foot mass reappeared with pain that was significantly worse while walking. In August 2019, due to the worsening of clinical symptoms, the patient transferred to our clinic for treatment from her original hospital, which kept her original medical examination and MRI files. Examination revealed swelling on the back of the left foot, surgical scars on the bottom of the foot, and a tender mass of about $10 \mathrm{~cm} \times 4 \mathrm{~cm}$. The patient experienced a decreased sensation on the lateral sides of the foot.

Color doppler ultrasound examination showed that there were multiple solid hypoechoic masses along the tibial nerve, as well as the nerve plantaris mediacis and lateralis, $9.4 \mathrm{~cm} \times 2.9 \mathrm{~cm} \times 1.5 \mathrm{~cm}$ in size (Figure 1). The tumor volume was calculated using the formula tumor length $\times$ width $\times$ height. The total tumor volume is the sum of each individual tumor's volume. Due to the failure of two prior surgical treatments during the early stages of the disease, the patient refused to undergo any further surgical treatments, including a biopsy of the recurrent tumor. After full discussion and communication with the patient and family, the patient agreed and signed the informed consent form to undergo sonodynamic therapy.

This patient had not undergone MRI examination during the consultation and entire treatment process. Throughout the course of treatment and postoperative follow-up observations, color doppler ultrasound was used to observe the patient's effect after treatment and tumor changes because clinically, color doppler ultrasound examinations are convenient, accurate, and cost-effective for neurofibrima examination of limbs. This reduces the patient's financial burden without impeding the examining doctor's ability to successfully diagnose and treat the illness, though in most cases, MRI imaging is the gold standard. 


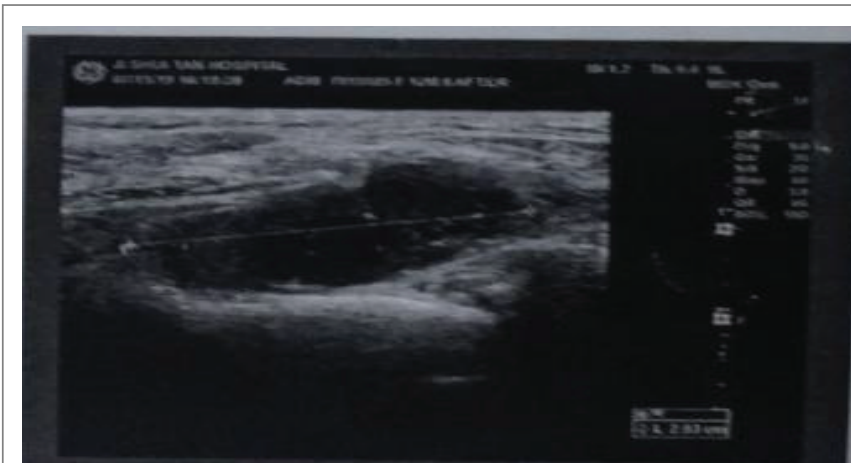

Figure 1: Pre-SDT, Tumor size: length $9.4 \mathrm{~cm} \times$ width $2.9 \mathrm{~cm} \times$ height $1.5 \mathrm{~cm}=40.89 \mathrm{~cm}^{3}$.

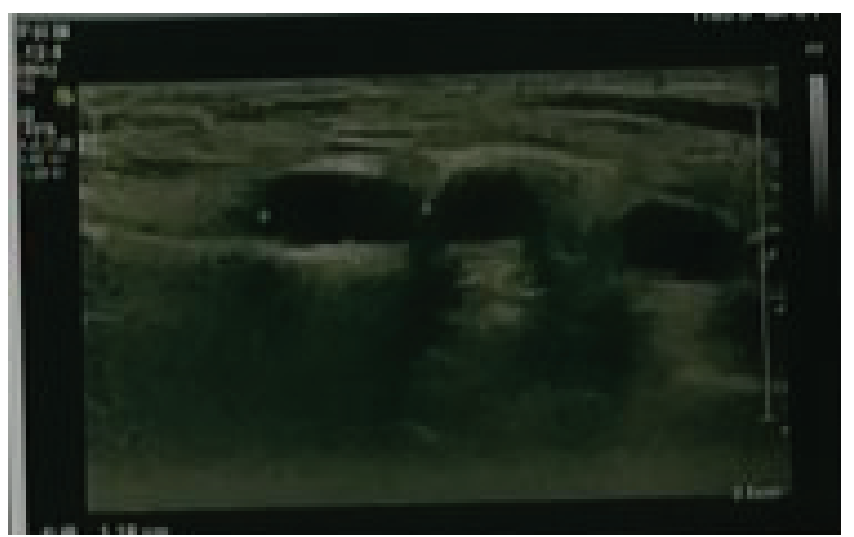

Figure 2: 10 months after SDT treatment, the total residual tumour volume is $9.1 \mathrm{~cm}^{3}+0.7 \mathrm{~cm}^{3}=9.8 \mathrm{~cm}^{3}$

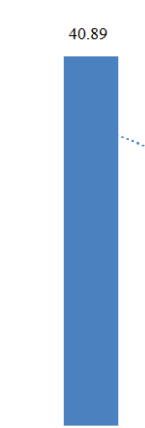

Time: before SDT

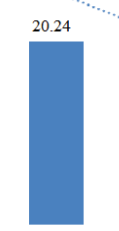

After SDT 1 mon

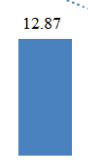

3 mon

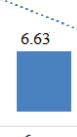

6 mon.

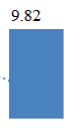

10 mon.
Figure 3: Tumor volume $\left(\mathrm{cm}^{3}\right)$ change after SDT.

After the skin test was confirmed negative for allergic reactions, a hematoporphyrin injection manufactured by HiPorfin, Chongqing Male Biopharmaceutical Co. Ltd. China, was intravenously injected at a dose of $5 \mathrm{mg} / \mathrm{kg}$ body weight. After having the patient stay away from light for 24 hours to ensure the effectiveness of the drug, the tumor was located using ultrasound equipment from SSD-a10 CUSG, Aloka, Japan and the XK-211HR tumor ultrasound treatment instrument manufactured by Wu Han Xin Kan Medical Instrument Co. China, was used to perform $3 \mathrm{D}$ omni directional acoustic dynamic treatment of the tumors in the left foot. The ultrasound frequency used for the treatment was $450 \mathrm{kHz}$ with intensity of $2 \mathrm{~W} / \mathrm{cm}^{2}$. Each treatment lasted 30 minutes, with two treatments per day, continuing for three days. On the second day of sonodynamic treatment, the swelling of the left foot disappeared detected by palpation and the patient reported significant reduction in pain while walking. Thirty days post-treatment, an ultrasound examination of the left foot showed that the tumor size was $8.8 \mathrm{~cm} \times 2.3 \mathrm{~cm} \times 1.0 \mathrm{~cm}$, a $50.5 \%$ reduction compared to the size prior to sonodynamic treatment. Other than occasional pinprick-like pike in the left foot in the three months after the procedure, the patient had no other abnormalities. A color doppler ultrasound examination of the left foot 10 months after sonodynamic treatment showed that the size of the subcutaneous hypoechoic mass was $8.2 \mathrm{~cm} \times 1.6 \mathrm{~cm}$ $\times 0.2 \mathrm{~cm}$ (Figure 2), an $88 \%$ tumor shrinkage compared to prior to sonodynamic treatment (Figure 1 and 3 ) and patient clinical physical examination was normal.

\section{Results and Discussion}

Neurofibromatosis is widely understood to be an autosomal dominant genetic disease. Neurofibromatosis type 1 (NF 1) is caused by a mutation in the tumor suppressor gene on chromosome 17q11.2, while Neurofibromatosis type 2 (NF 2) is due to mutations to the tumor suppressor gene in chromosome 22q12.2. The incidence rate of NF 1 is about $1 / 2500 \sim 3000$, of which $8-12 \%$ will become malignant $[1,2]$.

Plexiform neurofibroma accounts for about $30 \%$ of patients with NF1, caused mostly by genetic mutations. Superficial plexiform neurofibroma is often accompanied by hyperpigmentation and hypertrichosis. This type of tumor usually grows diffusely along the nerve trunk, with an appearance like "a bag of worms." Surgery is the only effective treatment for NF 1, but there are many disadvantages:

1) Surgery is unable to treat multiple neurofibromatosis

2) Tumor recurrence rate after surgery is high and the disease cannot be completely cured [3].

Sonodynamic therapy is likely to be a successful modality in the treatment of neurofibromatosis [1-3]. The drug used in sonodynamic therapy, the sensitizer hematoporphyrin, has a characteristic in that it is specifically taken in by tumor cells and not normal cells [4]. After tumor cells absorb the sensitizer, ultrasonic waves that do no damage to normal human biological tissues and have good penetration through bone and organs activate the sensitizer localized in tumor cells. This produces high concentrations of singlet oxygen ions and free radicals that can effectively destroy the organelles, cell membranes and DNA of tumor cells, thereby accurately killing tumor cells. Since normal tissue cells do not absorb the sensitizer, sonodynamic therapy does not damage normal tissue cells $[4,5]$.

The effectiveness of sonodynamic therapy in treating plexiform neurofibroma is highlighted in the case reveals that sonodynamic therapy is able to effectively control recurrent neurofibromas. Tumor necrosis shrunk by $>75 \% 10$ months after sonodynamic therapy and the patient's damaged nerve function fully recovered 3 months after treatment. The patient is currently under a 3 year-follow up regime post-SDT at an interval of 3 months.

\section{Conclusion}

The results of this treatment indicate that sonodynamic therapy is an effective, non-invasive, precise, and thorough treatment for plexiform neurofibroma. Continued clinical investigation of large-scale neurofibromatosis using sonodynamic therapy will determine how this technology can best be used in the treatment of neurofibromatosis and potentially create a new solution for this complex medical problem. 


\section{References}

1. Boyd KP, Korf BR, Theos A (2009) Neorufibromatosis type 1. J Am Acad Dermatol 61: 1-16.

2. Tchernev G, Chokoeva AA, Patterson JW, Bakardzhiev I, Wollina $U$, et al. (2016) Plexiform neurofibrama: A case report. Medicine (Baltimore) 95: e2663.

3. Antônio JR, Goloni-Bertollo EM, Trídico LA (2013) Neurofibromatosis: chronological history and current issues. An Bras Dermatol 88: 329343.

4. Rosenthal I, Sostaric JZ, Riesz P (2004) Sonadynamic therapy: a review of the synergistic effects of drugs and ultrasound. Ultrason Sonchem 11: 349-363.

5. Hirbe AC, Gutmann DH (2014) Neurofibromatosis type 1: a multidisciplinary approach to care. Lancet Neurol 13: 834-843. 